\title{
Development of Oxytocin- and Vasopressin-Network in the Supraoptic and Paraventricular Nuclei of Fetal Sheep
}

\author{
L. SHI ${ }^{* *}, Y$. FAN $^{2^{*}}, \mathrm{Z} . \mathrm{XU}^{1,3}$ \\ * These authors contributed equally to this work.
}

${ }^{1}$ Beijing Sport University and First Hospital of Soochow University, Beijing and Suzhou, China, ${ }^{2}$ Clinical Laboratory, Suzhou Kowloon Hospital Shanghai Jiao Tong University Medical School, Suzhou, China, ${ }^{3}$ Center for Perinatal Biology, Loma Linda University School of Medicine, Loma Linda, California, USA

Received September 12, 2011

Accepted March 2, 2012

On-line April 5, 2012

\section{Summary}

The hypothalamic supraoptic and paraventricular nuclei consist of oxytocin and arginine vasopressin synthesizing neurons that send projections to the neurohypophysis. A growing body of evidence in adult animals and young animals at near term confirmed the structure and function in the vasopressinergic and oxytocinergic network. However, whether those distinctive neural networks are formed before near term is largely unknown. This study determined the special patterns in location and distribution of oxytocin- and vasopressin-neurons in the paraventricular and supraoptic nuclei from preterm to term in the ovine fetuses. The results showed that oxytocin- and vasopressin-neurons were present in both nuclei at the three gestational time periods (preterm, near term, and term). In the paraventricular nuclei, vasopressin-cells concentrated mainly in the core of the middle magnocellular paraventricular nuclei, and oxytocin-cells were scattered surrounding the core. In the supraoptic nuclei, vasopressin-cells mostly located in the ventral part, and oxytocincells in the dorsal part. The data demonstrated that the special distributed patterns of vasopressin- and oxytocin-neuron network have formed in those two nuclei at least from preterm. Intracerebroventricular injection of angiotensin II significantly increased fetal plasma oxytocin and vasopressin levels at preterm, which was associated with an increase of oxytocin- and vasopressin-neuron activity marked with c-fos expression. The data provided new evidence for the structural and functional development of the oxytocin- and vasopressin-network before birth.

\section{Key words}

Distribution • Arginine vasopressin • Oxytocin • SON • PVN

\section{Corresponding author}

Zhice Xu, Center for Perinatal Biology, Loma Linda University School of Medicine, Loma Linda, California 92350, USA. E-mail: zxu@llu.edu

\section{Introduction}

The hypothalamus functions critically in a number of important physiological processes in the body, including cardiovascular regulation, body fluid homeostasis, stress responses, thermoregulation, appetite regulation, sexual behavior, and immune responses. Organizations of cellular networks in the brain can be determined by distinctive patterns of cell groups during development (Altman and Bayer 1986, Diepen 1962, Gilbert 1935, Keyser 1979, 1983, Kühlenbeck and Haymaker 1949). Arginine vasopressin (AVP) and oxytocin (OXT) are mainly synthesized in the magnocellular cells of the hypothalamic supraoptic and paraventricular nuclei (SON and PVN) whose axons project to the posterior pituitary (Brownstein et al. 1980). Upon appropriate stimulation such as hemorrhage, dehydration, central angiotensin II (Ang II) administration, these neurohypophysial hormones are released into blood stream to act on peripheral effectors (i.e., uterus, mammary myoepithelium, blood vessels, and kidney tubules etc.) (Acher and Chauvet 1995). 
It is not strange that appearance of certain physiological processes or brain functions is only accompanied with formation of special neuron networks in the brain. When would AVP or OXT neural networks in the hypothalamus form an adult pattern in anatomy during developmental periods is an interesting question. To answer such questions has become particularly important due to recently accumulated evidence for the hypothesis of adult diseases in developmental origins (Barker 1995, Godfrey and Barker 2001, KanakaGantenbein 2010).

Previous studies on rats have shown that AVP and OT receptors could be detected in the brain in embryonic day 12 (E12), and E14, respectively (Tribollet et al. 1989, 1991). Hoffman et al. also showed the immunocytochemical localization of OXT and AVP in the fetal sheep at near term and term (Hoffman et al. 1989). However, it is not clear about the distribution and functional capability of OXT-neurons at earlier gestational age. The present study was designed to determine the anatomical distribution of AVP- and OXTcontaining neurons in the SON and PVN from preterm to term in ovine fetuses, and to determine the responsiveness of these neurons in the fetal SON and PVN to central stimulation of Ang II.

\section{Methods}

Altogether nineteen-dated pregnant ewes (singleton) were used in the study. Among all the fetuses, nine (preterm, near term, term, each $n=3$ ) were used for the study of distribution patterns of AVP- and OXTneurons in the hypothalamus. The gestational age was $108 \pm 3$ days (preterm), 130 \pm 2 days (near term), and $145 \pm 2$ days (term), respectively. AVP (OXT)fluorescence immunostaining was carried out on these nine fetal brains. Another ten fetuses (preterm, 109 \pm 3 days gestation) were used for functional experiments of the AVP- and OXT-neurons. These ten fetal brains were used for double staining of FOS- and AVP (OXT)immunoreactivity (ir). All protocols in this study had been approved by the institutional animal care committee.

\section{Tissue collection}

The ewes were anesthetized under ketamine anesthesia $(20 \mathrm{mg} / \mathrm{kg} \mathrm{im})$ and ventilated with a mixture of isofluorane and oxygen. A midline abdominal incision was made, and the fetal head and neck were exposed. A 16-gauge needle was inserted into one side of the fetal carotid artery for perfusion. The fetus was perfused immediately following moving out of the utero via the carotid artery with $0.01 \mathrm{M}$ phosphate-buffered saline (PBS) followed by $4 \%$ paraformaldehyde (PFA) in $0.1 \mathrm{M}$ phosphate buffer under anesthesia. The perfusion period was $\sim 5-7 \mathrm{~min}$, and the perfusion volume of fixative was about $400-500 \mathrm{ml}$. The fetus was decapitated during perfusion. The brain was removed immediately following perfusion. Postfixation was performed in the same PFA solution for $12 \mathrm{~h}$, after which the brain was placed in $20 \%$ sucrose in $0.01 \mathrm{M}$ phosphate overnight. The ewes were sacrificed immediately following tissue collection.

\section{Immunostaining experiments}

$$
\text { AVP- / OXT-ir }
$$

As mentioned above, nine fetuses (preterm, near term, term, each $n=3$ ) were used for the study of distribution patterns of AVP- and OXT-neurons in the hypothalamus. Therefore, AVP (OXT) -fluorescence immunostaining was carried out on these fetal brains. Twenty-micrometer coronal sections of fetal brain were cut on a cryostat. PBS was used to store tissue sections. Every other section of the SON and PVN in the hypothalamus was used for examining the organization of AVP- and OXT-containing neurons. Immunocytochemical staining was performed as reported before (Xu et al. 2001). Briefly, tissues were incubated with $1 \%$ $\mathrm{H}_{2} \mathrm{O}_{2}$ for $30 \mathrm{~min}$ at room temperature with gentle agitation to block endogenous peroxidase activity. After blocking with $2 \%$ goat serum, the sections were incubated with the AVP or OXT antibody (1:5000, Diasorin, USA) overnight, and then anti-rabbit antibody (1:200, Vector Laboratories, USA) conjugated fluorescein-isothiocyanate was applied. All sections were mounted on slides, and then cover slipped. In this study, PVN and SON in the hypothalamus were evaluated from the same animal. All sliced and stained sections were analyzed. The sections were observed with a darkfield microscope with a filter for fluorescein-isothiocyanate excitation for incident light fluorescence. AVP- or OXTir staining was examined under Nikon Eclipse TE2000-U fluorescent microscopy.

\section{FOS-ir and AVP(OXT)-ir double staining}

At the end of the functional study (see next section), the fetal brains were removed and prepared immediately following the perfusion. Every other section 
of the SON and PVN in the hypothalamus was used for c-fos immunoreactivity (FOS-ir) staining. The tissue sections were incubated on a gentle shaker overnight at $4{ }^{\circ} \mathrm{C}$ in the primary antibody $(1: 15,000$, Santa Cruz Biotechnology, Santa Cruz, CA). The FOS primary antibody was raised from mouse. The sections were further incubated in anti-mouse serum (1:400) for $1 \mathrm{~h}$ and then revealed using fluorescein (Vector Laboratories). Immediately following FOS immunostaining, tissue sections for double immunostaining were incubated in the AVP or OXT antibody. Anti-rabbit antibody conjugated with a red fluorescent molecule was applied. The hypothalamic sections were also used for the control experiment in immunostaining. In which, all procedures were the same as described above, only the primary antibodies were not used. The results confirmed specificity of the antibodies used was the same as that of previously reported.

\section{Functional study of AVP- and OXT-neurons}

\section{Surgical preparations}

In order to investigate the development of functional capability in release of the hormones of AVPand OXT-neurons in response to central Ang II administration, ten time-dated pregnant ewes (109 \pm 3 days gestation) were used. Under the anesthesia, a midline abdominal incision was made to expose the uterus. In both the ewe and fetus, polyethylene catheters were placed in the maternal (inner diameter: $1.8 \mathrm{~mm}$; outer diameter: $2.3 \mathrm{~mm}$ ) and fetal (inner diameter: $1.0 \mathrm{~mm}$; outer diameter: $1.8 \mathrm{~mm}$ ) femoral vein and artery, and then were respectively threaded to the inferior vena cava and abdominal aorta. Intracranial cannula (18 gauge) was placed in the lateral ventricle and latency of the catheter at insertion was assessed by free flow of cerebral spinal fluid via gravity drainage (Shi et al. 2008). The fetus was then returned to the uterus, the hysterotomy was closed and all catheters were externalized to the maternal flank and placed in a cloth pouch. After surgery, pregnant ewes were returned to individual pens and allowed free access to food and water. Animals recovered for 4 5 days after surgery. Twice daily during the initial 2 days of recovery, antibiotics were administered intravenously daily to the ewe (70 mg gentamicin and $1 \mathrm{~g}$ oxacillin) and to the fetus (5 mg gentamicin and $30 \mathrm{mg}$ oxacillin).

\section{Physiological testing}

The experiments were performed on conscious, chronically instrumented fetal sheep, which were assigned into two groups randomly, control group $(n=5)$ and experimental group $(n=5)$. Studies lasted for 3 hours which included $1 \mathrm{~h}$-baseline period ( -60 to 0 minutes) and $2 \mathrm{~h}$-experimental period (0 to 120 minutes). Beginning at time 0, Ang II (1.5 $\mathrm{gg} / \mathrm{kg}$, Sigma $)$ in $0.9 \%$ $\mathrm{NaCl}(1 \mathrm{ml})$ was injected intracerebroventricularly into the fetus over 5 minutes. The dose of ANG II was chosen based on previously reported studies (Xu et al. 2001, Xu et al. 2005). Fetal body weight was estimated according to the following formula: fetal weight $(\mathrm{kg})=$ $0.0961 \times$ gestational age (days) $\times 9.228$ (Robillard et al . 1979). In the control group, $1 \mathrm{ml} 0.9 \% \mathrm{NaCl}$ (transport medium) was injected intracerebroventricularly into the fetuses. Throughout the study, maternal and fetal systolic and diastolic pressure and heart rate (HR) were monitored continuously. Fetal and maternal systolic (SP), diastolic pressure (DP), and HR were monitored using a Power Lab Physiological Record with Chart 5 software (AD Instruments). Maternal and fetal arterial blood was withdrawn at timed intervals for measurement of $\mathrm{pH}$, blood gases, hematocrit, plasma electrolyte composition, osmolality, AVP and OXT concentrations. The volume of blood sampled from the fetus was replaced immediately with an equivalent volume of heparinized maternal blood to avoid any hemodynamic effects of sampling. Blood $\mathrm{PO}_{2}, \mathrm{PCO}_{2}$, and $\mathrm{pH}$ were measured with a Nova analyzer Model pHOx Plus L (Nova Biochemical, Waltham, MA). Plasma osmolality was measured by freezing-point depression on an Advanced Digimatic Osmometer.

\section{Hormone experiments}

Measurements of AVP and OXT were performed by using radioimmunoassay (Shi et al. 2008). Blood samples were centrifuged immediately. The plasma was then stored at $-20{ }^{\circ} \mathrm{C}$ for radioimmunoassay. Plasma samples $(1.0 \mathrm{ml})$ were acidified with $1 \mathrm{~N} \mathrm{HCl}$ ( $0.15 \mathrm{ml}$ to each tube and mix thoroughly). AVP and OXT were extracted using Sep-Pak C18 cartridges (Waters Associates, Milford, MA). First, condition the C18 column by applying $10 \mathrm{ml}$ HPLC grade methanol to the column followed by $10 \mathrm{ml}$ of distilled water. Second, flush with $10 \mathrm{ml}$ of $0.1 \%$ TFA. Third, acidified plasma samples were added slowly to the columns, and the columns were washed with $0.1 \%$ trifluoroacetic acid (TFA) $10 \mathrm{ml}$. Then, the absorbed AVP and OXT were eluted with $50 \%$ methanol (HPLC grade) in $0.1 \%$ TFA, and the residues were dried in a Speed-Vac concentrator. 
The assay sensitivity was $1.25 \mathrm{pg}$ for AVP/tube and $1.62 \mathrm{pg}$ for OXT/tube. The intra- and inter-assay coefficients of variations were 7 and $9 \%$ for AVP, and 8 and $10 \%$ for OT, respectively. AVP and OXT recoveries average $70 \%$ in our laboratory. All plasma samples were processed together.

\section{Data analysis}

The number of FOS-ir positive cells in the SON and PVN was evaluated in a quantitive manner under a Nikon fluorescent microscopy directly. The labels on the glass slides were covered during counting, and the data from counting were treated in a blinded manner. The counting of positive c-fos staining was within the edges of the SON and PVN for all sections stained. All positive FOS-ir were counted. Colocalization of FOS-ir and AVPir in the same sections was also counted. SPSS software was used for statistical analysis. Group differences were evaluated by using MANOVA approaches to repeated measures. Comparison before and after the treatments was determined with $t$-test or one-way ANOVA followed by the Tukey post hoc test. Differences were considered significant when $\mathrm{P}<0.05$. All results are expressed as mean \pm SEM.
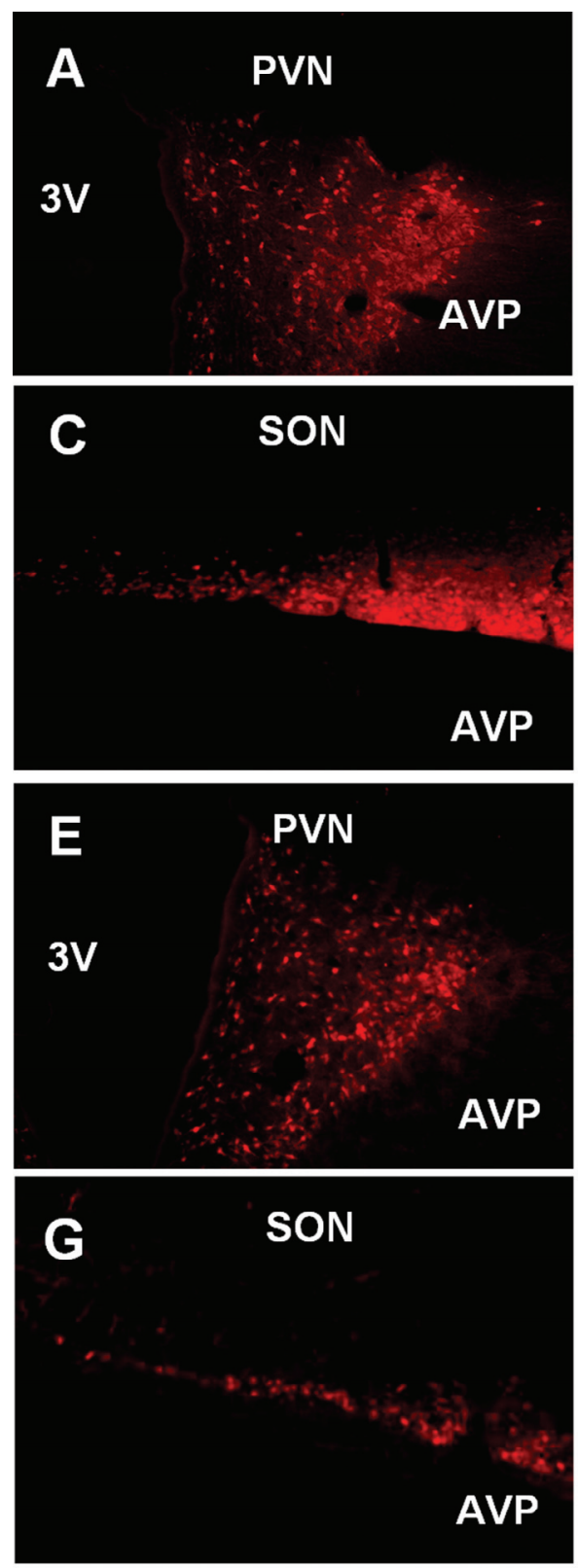
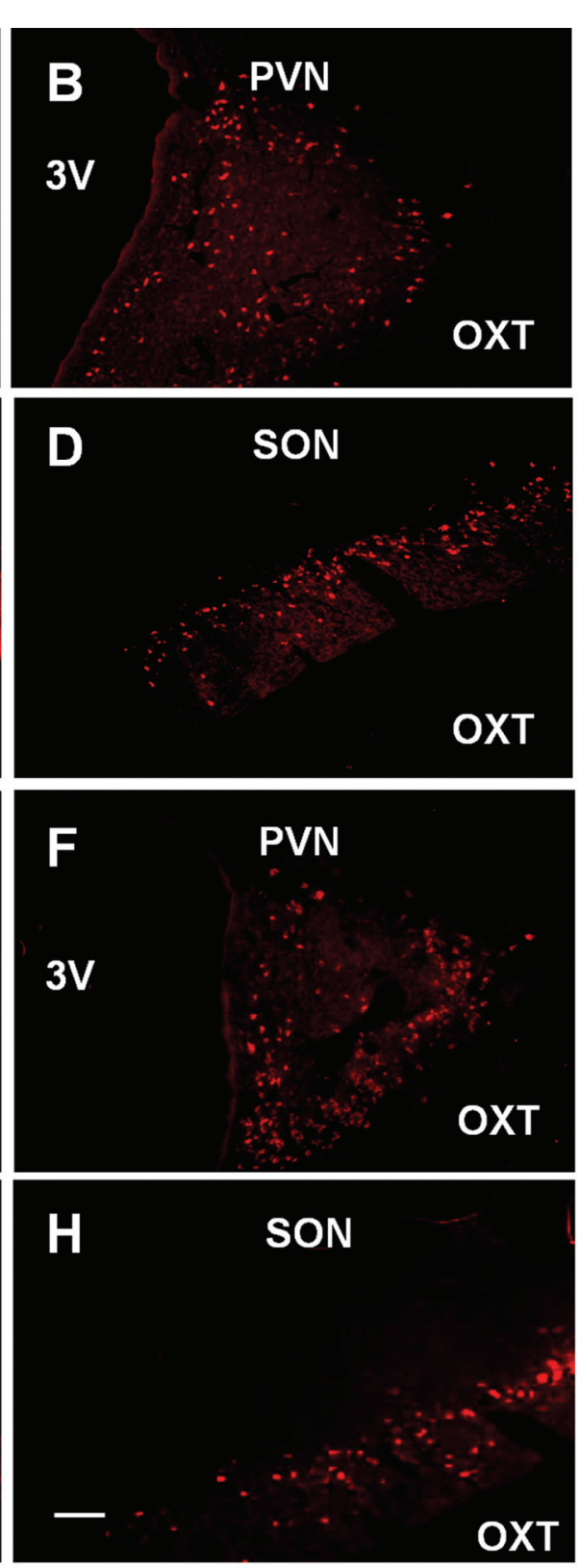

Fig. 1. Immunocytochemical staining of vasopressinergic and oxytocinergic neurons in the paraventricular nucleus (PVN) and the supraoptic nucleus (SON) at preterm and term. A-H are fluorescence pictures of AVP- or OXTimmunoreactive (ir) staining within the edges of PVN $(A / B, E / F)$ and SON $(C / D, G / H)$ observed under Nikon fluorescent microscopy. A-D: preterm ( $\sim 110 d$ gestation age). E-H: term ( $\sim 145 d$ gestation age). A, C, E, G: AVP-ir; B, D, F, H: OXT-ir. 3V, the third ventricle; AVP, arginine vasopressin; OXT, oxytocin. Scale bar $=200 \mu \mathrm{m}$. 


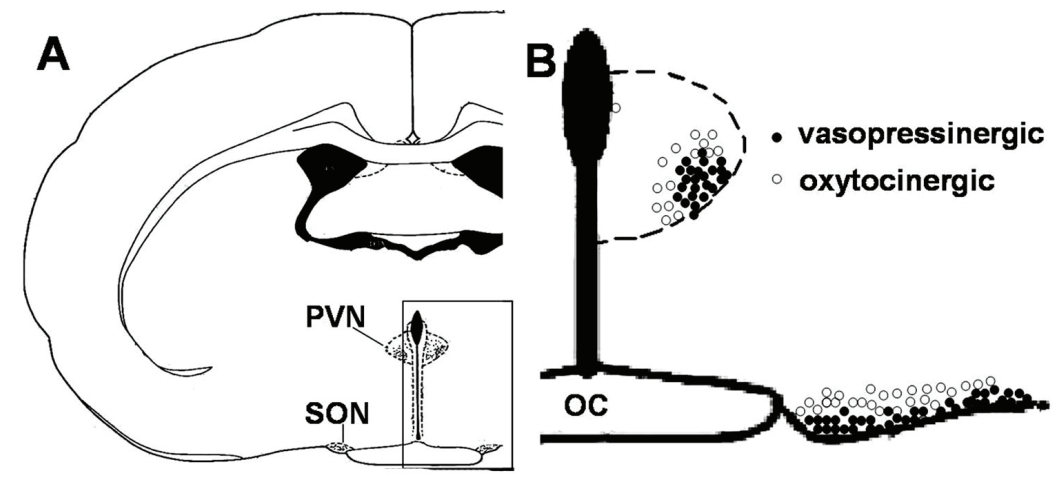

Fig. 2. The distribution of AVP- and OXT-ergic neurons in the PVN and SON of ovine fetuses at preterm. AVP, arginine vasopressin; OXT, oxytocin; $\mathrm{PVN}$, the paraventricular nucleus; SON, the supraoptic nucleus.

\section{Results}

Distribution patterns of AVP- and OXT-neurons in the fetal hypothalamus

At the three developing stages (preterm, nearterm, and term), immunocytochemistry showed AVP- and OXT-ir positive cells in both PVN and SON. Notably, distribution patterns of both AVP-stained and OXT-stained cells in the PVN and SON are similar at the preterm, nearterm, and term.

In the PVN, AVP-ir concentrated mainly in the middle of magnocellular part (Fig. 1A, E; Fig. 2), while OXT-ir were mainly scattered around the core of the PVN (Fig. 1B, F; Fig. 2). Such cellular distribution pattern is similar from preterm to term (Fig. 1A, B, E, F). In the SON, at the three gestational time, AVP-ir mostly concentrated at the ventral part (Fig. 1C, G; Fig. 2), while OXT-ir at the dorsal part of the SON (Fig. 1D, H; Fig. 2).

Neuroendocrine responses of AVP-and OXT-neurons to central Ang II administration

\section{Arterial blood values}

I.c.v. injection of Ang II $(1.5 \mu \mathrm{g} / \mathrm{kg})$ or transport medium had no effect on maternal or fetal plasma osmolality, $\mathrm{Na}^{+}, \mathrm{K}^{+}$, and $\mathrm{Cl}^{-}$concentrations, or arterial blood $\mathrm{pH}, \mathrm{PO}_{2}, \mathrm{PCO}_{2}$, hemoglobin, and hematocrit in either experimental or control group (all $P>0.05$ ). All arterial values were within normal ranges (all $P>0.05$ ).

There was no difference in maternal mean arterial pressure between the control and experimental groups $(P>0.05)$. Fetal mean arterial pressure $(P<0.01)$ was increased, and heart rate $(P<0.01)$ was decreased after i.c.v. administration of Ang II.

\section{Plasma AVP and OXT levels}

In both groups, i.c.v. injection of transport medium or Ang II did not change maternal plasma AVP and OXT levels significantly (data not shown). However, the fetal AVP and OXT concentrations were significantly increased after i.c.v. injection of Ang II (AVP: $\mathrm{F}_{8,1}=58.50$, OXT: $\mathrm{F}_{8,1}=35.38, P<0.01$ ) (Fig. 3). Fetal plasma AVP and OXT increased significantly within 15 minutes after i.c.v. Ang II administration, and the peak levels of plasma AVP and OXT were observed at 30 minutes after Ang II injection.

A

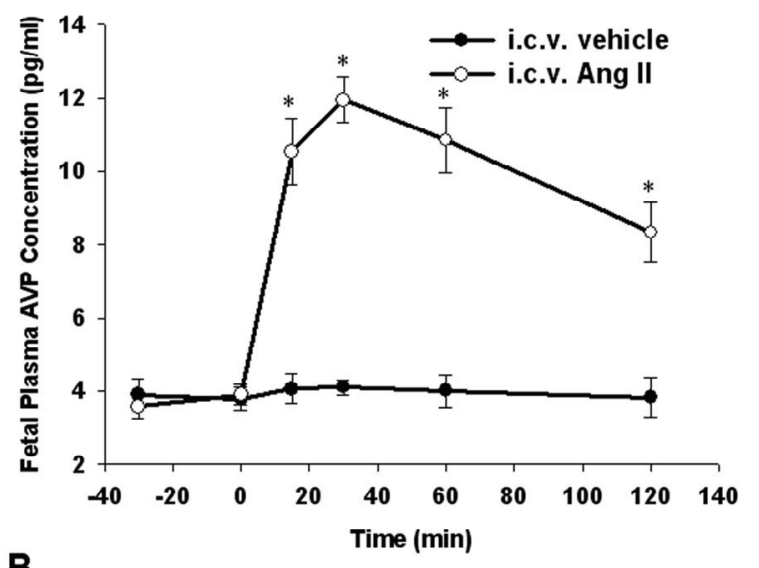

B

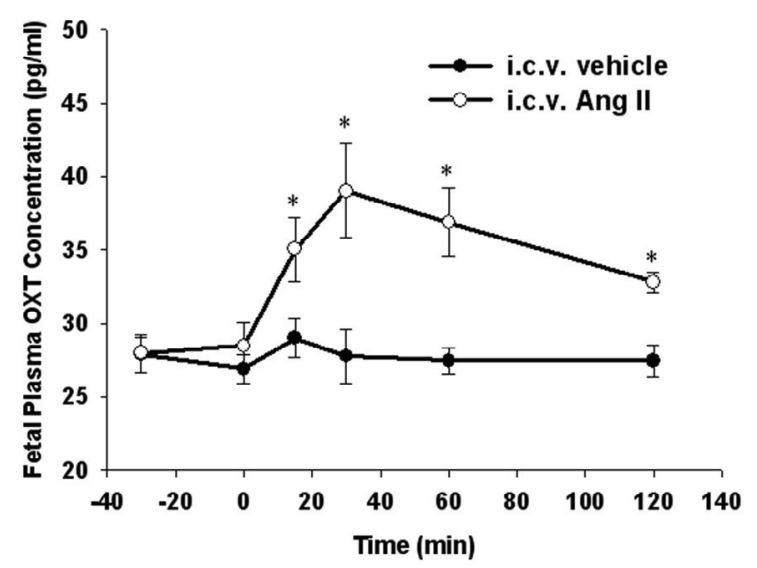

Fig. 3. The effect of i.c.v. Ang II on fetal plasma AVP (A) and OXT (B) concentration at preterm. The dose of Ang II, $1.5 \mu \mathrm{g} / \mathrm{kg} ; 0 \mathrm{~min}$ : time for i.c.v. injection. $* \mathrm{P}<0.01$ compared with the baseline level. $\mathrm{N}=5$ in each group. Ang II, angiotensin II; AVP, arginine vasopressin; OXT, oxytocin; PVN, the paraventricular nucleus; SON, the supraoptic nucleus. 


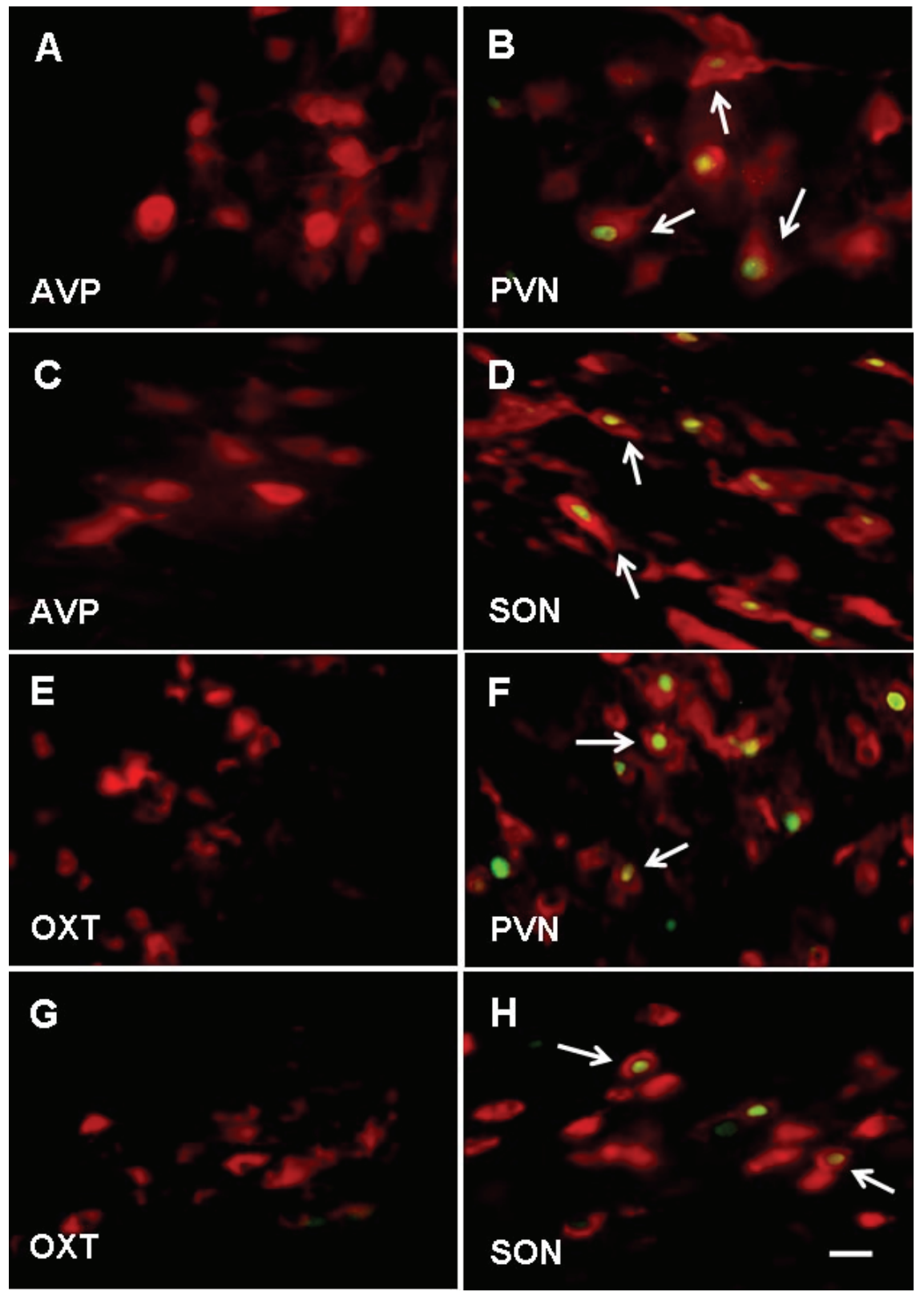

Fig. 4. FOS-ir and AVP-ir (or OXT-ir) in the PVN and SON following i.c.v. Ang II. $A / B$ and $E / F$ : double labeling of FOS-ir and AVP-ir in the PVN (A/B) and SON (E/F). C/D and $G / H$ : double labeling of FOS-ir and OXT-ir in the PVN (C/D) and SON $(G / H)$. Arrows indicate co-localization of FOS-ir and AVP-ir (or OXT-ir). Scale bar $=30 \mu \mathrm{m}$. Green represents FOS-ir positive, and red represents AVP-ir positive. Ang II, angiotensin II; AVP, arginine vasopressin; ir: immunoreactivity; OXT, oxytocin; $\mathrm{PVN}$, the paraventricular nucleus; SON, the supraoptic nucleus.
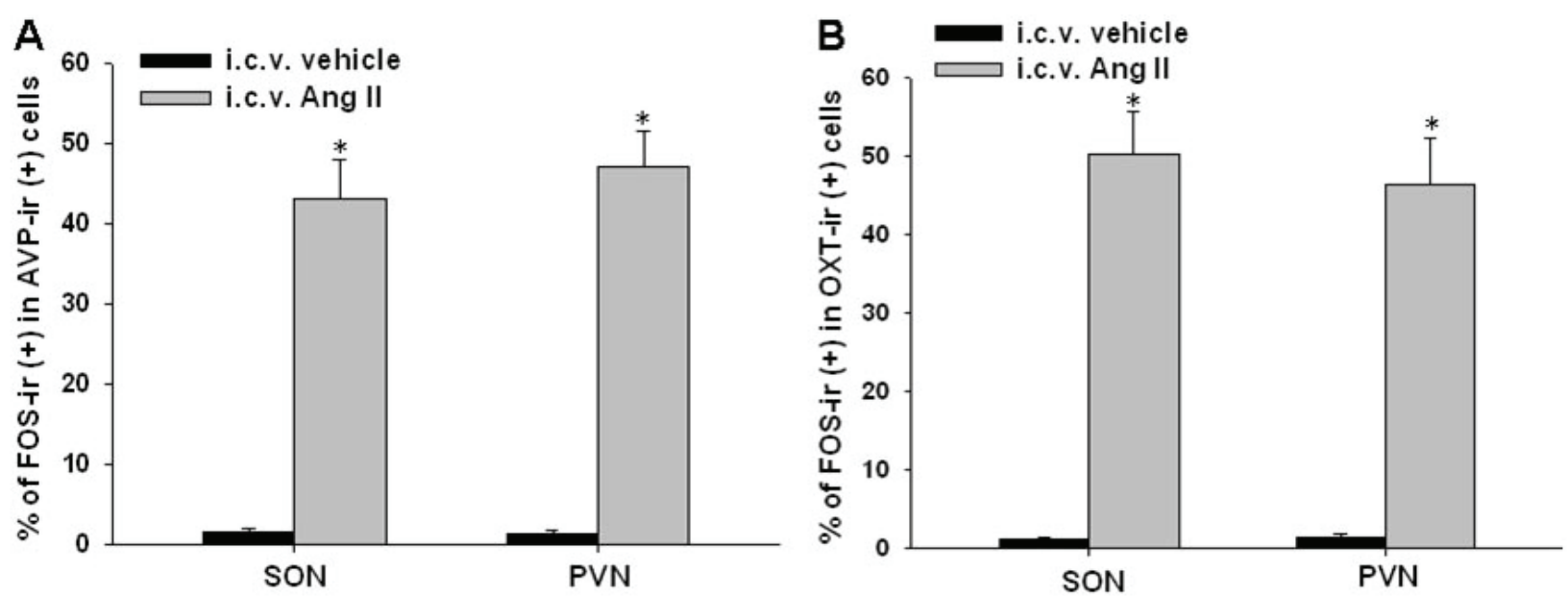

Fig. 5. Percentage of FOS-ir (+) in AVP-ir (+) / OXT-ir (+) cells in the fetal hypothalamus at preterm. A: i.c.v. vehicle; B: i.c.v. Ang II $(1.5 \mu \mathrm{g} / \mathrm{kg})$. Ang II, angiotensin II; AVP, arginine vasopressin; ir: immunoreactivity; OXT, oxytocin; PVN, the paraventricular nucleus; SON, the supraoptic nucleus. (+): positive. $* \mathrm{P}<0.01$ compared with the control level. 


\section{FOS-ir and double labeling}

In the control preterm fetuses, there was few FOS-ir in the PVN and SON. However, i.c.v. injection of Ang II produced intense FOS-ir in these structures in the fetal hypothalamus. There was significant difference of FOS-ir in the SON and PVN between the i.c.v. transport medium and the i.c.v. Ang II injected fetuses ( $\mathrm{t}=8.4$ and 10.9 , respectively, both $P<0.01$ ). Double labeling showed that there was no FOS-ir in AVP-containing and OXTcontaining cells in the control fetuses at preterm. However, colocalization of FOS-ir in AVP and OXT neurons was detected in both sides of the SON and PVN in the fetal hypothalamus following i.c.v. Ang II (Fig. 4). There was FOS-ir observed in none-VP or none-OT neurons. There was no difference in the total counting of AVP-ir in the SON and PVN between the control and the treated fetuses (all $P>0.05$ ). Double labeling of FOS-ir and AVP-ir / OXT-ir in the SON (43.2 $\pm 4.8 \%$ / $50.3 \pm 5.4 \%)$ and PVN (47.2 $\pm 4.4 \% / 46.3 \pm 6.1 \%)$ was significantly higher in the Ang II-treated fetuses than that in the control $(1.5 \pm 0.5 / 1.2 \pm 0.1 \%$ and $1.3 \pm 0.4 \%$ / $1.5 \pm 0.4 \%$, respectively) $(\mathrm{t}=6.5 / 8.2$ and $7.8 / 7.4$, respectively, $P<0.01$ ) (Fig. 5). It was also observed stimulated FOS-ir localized in none-AVP or none-OT cells. In SON, FOS-ir localized in none-AVP/none-OXT cells was $20 \pm 2.4 \% / 72 \pm 6.5 \%$. In PVN, FOS-ir localized in none-AVP/none-OXT cells was $63 \pm 7.1 \% / 23 \pm 3.3 \%$.

\section{Discussion}

The present study provides new information that the structural organization of OXT-neurons in the PVN and SON in the fetuses shows similar patterns to that in the adults at least from preterm. In addition, OXT- and AVP-neurons are functional at least from preterm in response to central Ang II stimulation.

The role of magnocellular neurosecretory neurons in the synthesis, transport, and release of the posterior pituitary hormones AVP and OXT is welldocumented in adults (Brownstein et al. 1980). The SON and PVN are main sources for AVP and OXT hormones released into the peripheral circulation. Immunohistochemical studies have clarified the organization of OXT- and AVP-ir cells in different species (Dierickx 1980). Neurons for those two peptides have been localized primarily in the PVN and the SON.

In the SON, previous studies showed that OXTpositive cells mainly concentrated dorsally, while AVPpositive cells located ventrally (McNeill and Sladek
1980, Rhodes et al. 1981, Sokol et al. 1976, Swaab et al. 1975b, Swanson et al. 1981, Vandesance and Dierickx 1975). Estimates of the ratio of AVP- and OXT-stained cells in the SON range from 1:1 (Vandesande and Dierickx 1975) to $1.6: 1$ (Swaab et al. 1975a, b) in rats. The organization of OXT- and AVP-ir network in the PVN is no less distinctive. At the magnocellular part of the nucleus, AVP-ir neurons are mainly located in the core, while OXT-ir neurons are primarily distributed around the core in the hypothalamus of adults (Rhodes et al. 1981, Sawchenko and Swanson 1982).

In fetal studies, Hoffman et al. demonstrated the AVP- and OXT-containing neurons in the PVN and SON in sheep before birth from the near term. In the present study, we also used preterm fetuses in study of formation of cellular network in those two nuclei. In our analysis, the similar distribution patterns of AVP and OXT and the PVN and SON were observed and noted. This indicated that distribution patterns of this cell network in the fetal hypothalamus appeared at least at preterm, providing new information on the anatomical development of those networks in the fetus. Our results not only confirmed the previous study by Hoffman and others at the near-term for both OXT- and AVP-ir neuron networks (Hoffman et al. 1989), but also demonstrated new findings at the preterm, indicating that the cellular networks for OXT-ir neurons in the SON and PVN have been well developed in the ovine brain at $70 \%$ gestation. In addition, our data confirmed that AVP-ir neuron network at the preterm was the same as that in the previous reports. This also suggested that physiological functions based on those two peptide networks in the hypothalamus may start at that gestational and developmental time.

The neuroendocrine experiments of AVP- and OXT-ir neurons in response to central Ang II administration provided new functional evidence. In the fetuses at preterm, i.c.v. injection of Ang II induced a marked increase of fetal plasma OXT and AVP concentrations without influence on maternal neurohypophysial hormone levels. The data about i.c.v. Ang II-induced AVP release in the present study was the similar to what reported before (Xu et al. 2005). The fetal OXT and AVP in circulation reached the peak in $30 \mathrm{~min}$. The increase of OXT and AVP lasted for more than $120 \mathrm{~min}$, which was associated with an increase of OXTand AVP-ir neuron activity marked with c-fos expression in the fetal hypothalamus. In the present study, we also observed stimulated FOS-ir localized in none-VP or none-OT cells. Whether those cells were stress-related or 
other function-related neurons need to be further studied. Accumulating evidence has shown that various signals, including Ang II, hypoxemia, and osmotic and hypovolemic stimulation, can induce AVP and OT production and release from the hypothalamus and pituitary (Brimble et al. 1978, Gutkowska et al. 2000, Ross et al. 1986, Share 1988, Stegner et al. 1984). In consistent with our previous studies (Xu et al. 2004, 2005), the fetal physiological status remained stable after surgical recovery and under the condition of i.c.v. injection of Ang II. The arterial values $\left(\mathrm{pH}, \mathrm{PCO}_{2}, \mathrm{PO}_{2}\right.$, hematocrit, hemoglobin, and plasma $\mathrm{Na}^{+}$) were not changed. Thus, we can exclude the possibility that the fetal AVP and OT release was due to hypoxemia, osmotic or hypovolemic factors. A more likely explanation for the elevation of the preterm fetal plasma AVP and OT is the facilitation by central Ang II stimuli. As mentioned above, AVP and OXT are mainly synthesized in the magnocellular cells of the hypothalamic SON and PVN (Brownstein et al. 1980). Our and others' previous studies demonstrated rich and intensive angiotensin receptors in both SON and PVN (Hu et al. 2004, Wright and Harding 1995). Whether directly or indirectly actions of Ang II on AVP and OXT neurons in the SON and PVN deserves further study. Nevertheless, the present study showed activation of AVP and OXT cells in those nucleus accompanied with an increase of release of AVP and OXT provided interesting information on fetal development of the neuropeptide network in the hypothalamus. In addition, as shown in Fig. 5, it was also observed stimulated FOS-ir localized in none-AVP or none-OT cells following i.c.v. Ang II. Although this study focused on AVP and OXT cells only, it is worth for future investigation to further characterizing those cells.

The importance of study of the structural and functional development of neuropeptide systems in the fetal hypothalamus around preterm period in fetuses are linked to recent progress in demonstration of sensitive "window" time in fetal life for development of adult diseases (Nuyt and Alexander 2009). Therefore, the present study not only confirmed the structural networks of AVP- and OXT-systems in the SON and PVN at near term and term in fetal sheep, but also demonstrated novel finding that appearance of specific structural OXT pattern in the SON and PVN at preterm. Since AVP data was similar to what reported before (Hoffman et al. 1989, Xu et al. 2005), the major novel finding in this paper is the data about OXT at the preterm, especially its specific distribution patterns in the PVN and SON at that time point during fetal brain development. In addition, the functional studies showed OXT-containing neurons in the fetal hypothalamus could be activated by Ang II stimulation at preterm time.

In summary, the structural organization of OXTand AVP-ir neurons in the PVN and SON in the fetuses showed similar patterns to that in the adults at least from preterm. The findings of the present study provide new evidence for structural and functional development of the neuropeptide networks in the fetal hypothalamus at preterm. The information obtained will help us to understand the development of OXT and AVP neuron network in the fetal brain from preterm.

\section{Conflict of Interest}

There is no conflict of interest.

\section{Acknowledgements}

Supported by HL600355 and 2012CB947600, Nature \& Science Foundation (31071033, 81030006, 81070540), Training program Foundation for Beijing Talents (2010D009010000001) and Scientific Research Foundation for the Returned Overseas Chinese Scholars, Research Fund for the Doctoral Program of Higher Education of China (20101112120001). We thank Mr. Richard Wang (Cambridge, U.K.) for his helping in English.

\section{References}

ACHER R, CHAUVET J: The neurohypophysial endocrine regulatory cascade: precursors, mediators, receptors, and effectors. Front Neuroendocrinol 16: 237-289, 1995.

ALTMAN J, BAYER SA: The development of the rat hypothalamus. Advances in Anatomy Embryology and Cell Biology, Vol. 100. Springer-Verlag, Berlin, 1986.

BARKER DJP: Fetal origins of coronary heart disease. BMJ 311: 171-174, 1995.

BRIMBLE MJ, DYBALL REJ, FORSLING ML: Oxytocin release following osmotic activation of oxytocin neurones in the paraventricular and supraoptic nuclei. $J$ Physiol 278: 69-78, 1978. 
BROWNSTEIN MJ, RUSSELL JT, GAINER H: Synthesis, transport, and release of posterior pituitary hormones. Science 207: 373-378, 1980.

DIEPEN R: Der Hypothalamus. In: Handbuch der Mikroskopischen Anatomie des Menschen, vol IV/7. VON MÖLLENDORFF W, BERGMANN W. (eds), Springer, Berlin, pp. 1-525, 1962.

DIERICKX K: Immunocytochemical localization of the vertebrate cyclic nonapeptide neurohypophyeal hormones and neurophysins. Int Rev Cytol 62: 119-185, 1980.

GILBERT MS: The early development of the human diencephalon. J Comp Neurol 62: 81-116, 1935.

GODFREY KM, BARKER DJP: Fetal programming and adult health. Public Health Nutr 4: 611-624, 2001.

GUTKOWSKA J, JANKOWSKI M, MUKADDAM-DAHER S, MCCANN SM: Oxytocin is a cardiovascular hormone. Braz J Med Biol Res 33: 625-633, 2000.

HOFFMAN GE, MCDONALD T, FIGUEROA JP, NATHANIELSZ PW: Neuropeptide cells and fibers in the hypothalamus and pituitary of the fetal sheep: comparison of oxytocin and arginine vasopressin. Neuroendocrinology 50: 633-643, 1989.

HU F, MORRISSEYA P, YAO J, XU Z: Development of AT1 and AT2 receptors in the ovine fetal brain. Dev Brain Res 150: 51-61, 2004.

KANAKA-GANTENBEIN C: Fetal origins of adult diabetes. Ann N Y Acad Sci 1205, 99-105, 2010.

KEYSER A: Development of the hypothalamus in mammals. In: Anatomy of the Hypothalamus. MORGANE PJ, PANKSEPP J (eds), Marcel Dekker, New York, 1979, pp. 65-136.

KEYSER A: Basic aspects of development and maturation of the brain: embryological contributions to neuroendocrinology. Psychoneuroendocrinology 8: 157-181, 1983.

KÜHLENBECK H, HAYMAKER W: The derivatives of the hypothalamus in the human brain; their relation to the extrapyramdal and autonomic systems. Military Surg 105: 26-52, 1949.

MCNEILL TH, SLADEK JR Jr: Simultaneous monoamine histofluorescence and neuropeptide immunocytochemistry. II. Correlative distribution of catecholamine varicosities and magnocellular neurosecretory neurons in the rat supraoptic and paraventricular nuclei. J Comp Neurol 193: 1023-1033, 1980.

NUYT AM, ALEXANDER BT: Developmental programming and hypertension. Curr Opin Nephrol Hypertens 18: 144-152, 2009.

RHODES CH, MORRELL JI, PFAFF DW: Immunohistochemical analysis of magnocellular elements in rat hypothalamus: Distribution and numbers of cells containing neurophysin, oxytocin, and vasopressin. J Comp Neurol 198: 45-64, 1981.

ROBILLARD JE, MATSON JR, SESSIONS C, SMITH FG: Development aspects of renal tubular reabsorption of water in the lamb fetus. Pediatr Res 13: 1172-1176, 1979.

ROSS MG, EVIN MG, LEAKE RD, HUMME JA, FISHER DA: Continuous ovine fetal hemorrhage: sensitivity of plasma and urine arginine vasopressin response. Am J Physiol 251: E464-E469, 1986.

SAWCHENKO PE, SWANSON LW: Immunohistochemical identification of neurons in the paraventricular nucleus of the hypothalamus that project to the medulla or to the spinal cord in the rat. $J$ Comp Neurol 205: 260-272, 1982.

SHARE L: Role of vasopressin in cardiovascular regulation. Physiol Rev 68: 1248-1284, 1988.

SHI L, MAO C, ZENG F, ZHANG Y, XU Z: Central cholinergic signal-mediated neuroendocrine regulation of vasopressin and oxytocin in ovine fetuses. BMC Dev Biol 8: 95, 2008.

SOKOL HW, ZIMMERMAN EA, SAWYER WH, ROBINSON AG: The hypothalamo-neurohypophyseal system of the rat: Localization and quantitation of neurophysin by light microscopic immunocytochemistry in Brattleboro rats deficient in vasopressin and a neurophysin. Endocrinology 98: 1176-1188, 1976.

STEGNER H, LEAKE RD, PALMER SM, OAKES G., FISHER DA: The effect of hypoxia on neurohypophysial hormone release in fetal and maternal sheep. Pediatr Res 18: 188-191, 1984.

SWAAB DF, NIJVELDT F, POOL CW: Distribution of oxytocin and vasopressin cells in the rat supraoptic and paraventricular nucleus. $J$ Endocrinol 67: 461-462, 1975a.

SWAAB DF, POOL CW, NIJVELDT F: Immunofluorescence of vasopressin and oxytocin in the rat hypothalamoneurohypophyseal system. J Neural Trans 36: 195-215, 1975 b. 
SWANSON LW, SAWCHENKO PE, BÉROD A, HARTMAN BK, HELLE KB, VANORDEN DE: An immunohistochemical study of the organization of catecholaminergic cells and terminal fields in the paraventricular and supraoptic nuclei of the hypothalamus. J Comp Neurol 196: 271-285, 1981.

TRIBOLLET E, CHARPAK S, SCHMIDT A, DUBOIS-DAUPHIN M, DREIFUSS JJ: Appearance and transient expression of oxytocin receptors in fetal, infant, and peripubertal rat brain studied by autoradiography and electrophysiology. J Neurosci 9: 1764-1773, 1989.

TRIBOLLET E, GOUMAZ M, RAGGENBASS M, DUBOIS-DAUPHIN M, DREIFUSS JJ: Early appearance and transient expression of vasopressin receptors in the brain of rat fetus and infant. An autoradiographical and electrophysiological study. Brain Res Dev Brain Res 58: 13-24, 1991.

VANDESANDE F, DIERICKX K: Identification of the vasopressin producing and of the oxytocin producing neurons in the hypothalamic magnocellular neurosecretory system of the rat. Cell Tissue Res 164: 153-162, 1975.

WRIGHT JW, HARDING JW: Brain angiotensin subtypes AT1, AT2, and AT4 and their functions. Regul Pept 59: 269-295, 1995.

XU Z, CALVARIO G, YAO J, DAY L, ROSS MG: Central angiotensin induction of fetal brain c-fos expression and swallowing activity. Am J Physiol Regul Integr Comp Physiol 280: R1837-R1843, 2001.

XU Z, HU F, SHI L, SUN W, WU J, MORRISSEY P, YAO J: Angiotensin-induced vasopressin release and activation of hypothalamic neuron in pre-term fetuses. Peptides 26: 307-314, 2005.

XU Z, SHI L, YAO J: Central angiotensin II-induced pressor responses and neural activity in utero and hypothalamic angiotensin receptors in preterm ovine fetus. Am J Physiol Heart Circ Physiol 286: H1507-H1514, 2004. 